\title{
Technical Report: A Cost-Effective, Easily Available Tofu Model for Training Residents in Ultrasound-Guided Fine Needle Thyroid Nodule Targeting Punctures
}

\author{
Yun-Fei Zhang, $\mathrm{PhD}^{1}$, Hong Li, $\mathrm{PhD}^{2}$, Xue-Mei Wang, $\mathrm{PhD}^{1}$ \\ Departments of ${ }^{1}$ Ultrasound and ${ }^{2}$ Transplantation and General Surgery, The First Hospital of China Medical University, Shenyang, China
}

Objective: To establish a cost-effective and easily available phantom for training residents in ultrasound-guided fine needle thyroid nodule targeting punctures.

Materials and Methods: Tofu, drinking straws filled with coupling gel, a urine tube, and 21-gauge needles were used to generate a phantom thyroid with nodules for training. Twelve radiology residents were involved in the study. The puncture success rates were recorded and compared before and after phantom training using the Wilcoxon signed-rank test.

Results: On ultrasonography, tofu mimicked the texture of the thyroid. Drinking straws filled with coupling gel mimicked vessels. The urine tube filled with air mimicked the trachea, and 21-gauge needles mimicked small nodules in the transverse section. The entire phantom was similar to the structure of the thyroid and surrounding tissues. The puncture success rates of radiology residents were significantly increased from $34.4 \pm 14.2 \%$ to $66.7 \pm 19.5 \%$ after training $(p=0.003)$. The phantom was constructed in approximately 10 minutes and materials cost less than CNY 10 (approximately \$1.5) at a local store.

Conclusion: The tofu model was cost-effective, easily attainable, and effective for training residents in ultrasound-guided fine needle thyroid nodule targeting punctures in vitro.

Keywords: Ultrasound-guided; Fine needle aspiration biopsy; Thyroid nodules; Phantoms

\section{INTRODUCTION}

With the increasing incidence of thyroid carcinomas, the management of thyroid nodules has become controversial (1). Ultrasound-guided fine needle aspiration biopsy (FNAB) plays a crucial role in the management of thyroid nodules due to the safety and accuracy of this procedure (2). Before performing FNAB on patients, it is advisable to first practice on phantoms. Numerous types of training phantoms have been reported for breast biopsy (3-5); however, few FNAB

Received December 22, 2017; accepted after revision May 9, 2018. Corresponding author: Xue-Mei Wang, PhD, Department of Ultrasound, The First Hospital of China Medical University, No. 155 Nanjing North Street, Shenyang 110001, China.

- Tel: (86) 2483282098 - Fax: (86) 2483282997

- E-mail: wxmcmu@163.com

This is an Open Access article distributed under the terms of the Creative Commons Attribution Non-Commercial License (https://creativecommons.org/licenses/by-nc/4.0) which permits unrestricted non-commercial use, distribution, and reproduction in any medium, provided the original work is properly cited. models for thyroid have been reported. The ideal phantom is inexpensive, easily available, and has a consistency similar to that of thyroid tissue. In 2014, Abraham (6) described a novel phantom suspension system using super-concentrated gelatin for ultrasound-guided thyroid FNAB training. This model served as a practical training phantom that was quite useful before operating on patients. However, phantom training models that are cheaper, more readily available, and of similar consistency to the thyroid and surrounding structures are needed. Therefore, the purposes of our study were to construct a cost-effective and easily attainable training phantom for ultrasound-guided fine needle thyroid nodule targeting punctures and to evaluate its usefulness for puncture training.

\section{MATERIALS AND METHODS}

Tofu (also known as bean curd), drinking straws, a urine tube, and 21-gauge needles were used to construct the phantom (Fig. 1). The tofu was used to mimic the thyroid 


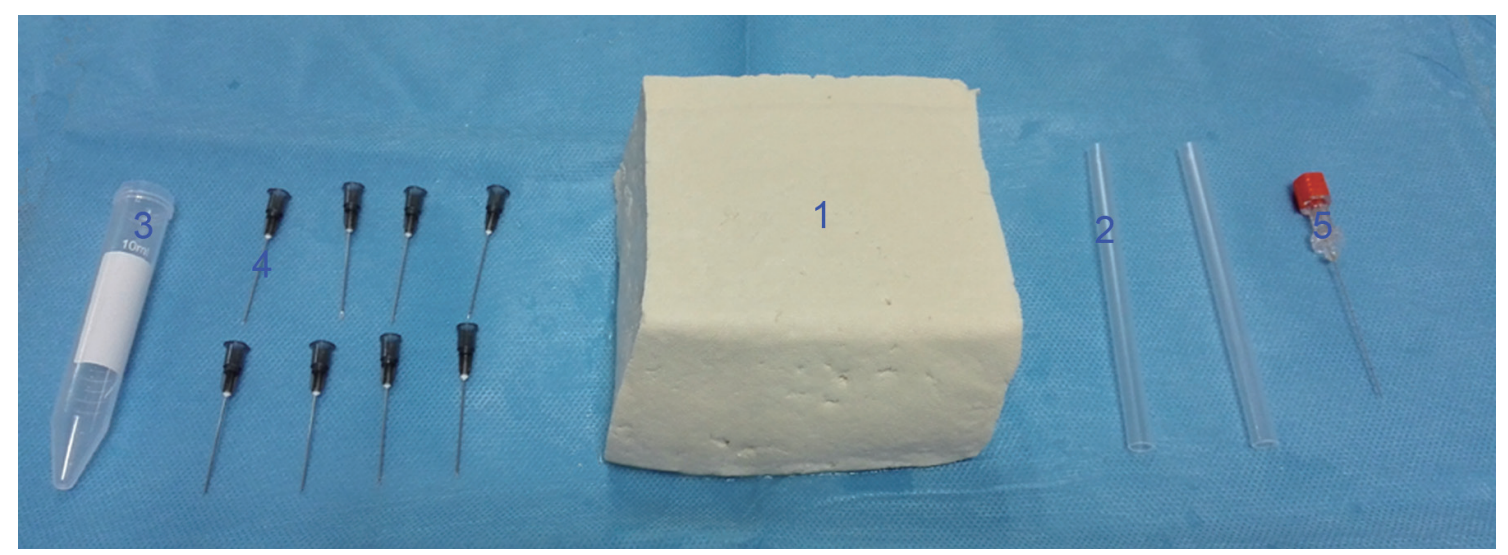

Fig. 1. Preparation of thyroid phantom for fine needle puncture. 1, Tofu; 2, drinking straw; 3, urine tube; 4, 21-gauge needle; 5 , 25-gauge puncture needle.

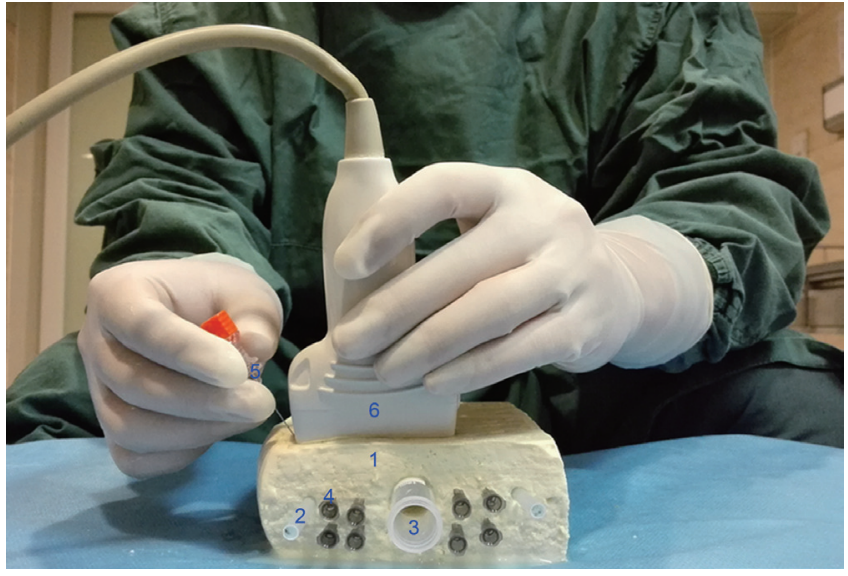

Fig. 2. Thyroid phantom. 1, Tofu; 2, drinking straw; 3, urine tube; 4, 21-gauge needle; 5, 25-gauge puncture needle; 6 , probe.

parenchyma. Drinking straws filled with coupling gel were used to mimic blood vessels. A 10-mL urine tube filled with air was used to mimic the trachea.

First, to produce the phantom, the tofu center was gently punctured with a urine tube, taking care to avoid rupture. Then, two drinking straws filled with coupling gel were inserted into the tofu parallel to the urine tube on either side, approximately $2 \mathrm{~cm}$ from the lateral side of the urine tube. Finally, eight 21-gauge needles (outer diameter of 0.81 $\mathrm{mm}$ ) were used to puncture the tofu parallel to the "vessels" and "trachea" to mimic "small nodules" in the "thyroid." The needles were distributed evenly in four different quadrants of dual "thyroid lobes" between the "vessel" and "trachea." The cost of materials for the phantom was calculated. Preparation time was also recorded. A linear transducer (ultrasound machine: HI VISION Avius, Hitachi Ltd., Tokyo, Japan) was used to guide the needle puncture in the "transverse" section of the "thyroid" (Fig. 2). A 25-gauge needle was used for ultrasound-guided fine needle punctures.

During a scheduled simulation session, 12 radiology residents from postgraduate years 2 and 3 were introduced to the phantom model, and the "trachea," "vessels" and each "small nodule" were identified. None of the residents had prior FNAB training or experience. After a demonstration without practice, they were asked to puncture each of the eight "small nodules" using a 25-gauge needle. Two doctors with more than 3 years of experience in FNAB judged whether the procedure was successful. One observed whether the 25-gauge needle touched the "small nodule" on the screen while avoiding the "trachea" and "vessels." The other observed for movement of the 21-gauge needle hub outside of the tofu. Success was achieved when the 25-gauge puncture needle touched the "small nodule" (modeled by a 21-gauge needle), without injuring the "trachea" and "vessels," and the 21-gauge needle hub outside the tofu moved. Different needle inserting traces were scheduled in order to practice alternating using one hand to handle the transducer and the other to hold the needle. Both superficial and deep targets were punctured (Fig. 3). All of the residents operated on the same phantom. Puncture success for each of the eight "small nodules" on the first attempt was recorded before training. Each resident was then given 30 minutes to practice on other similar, phantoms. Following this, the residents were asked to puncture the nodules on the same pre-training phantom, and each resident's puncture success rate for targeting the eight "small nodules" was recorded. In addition, puncture success rates of right-handed needling (Fig. 3; attempt b, c, $f, g$ ) versus left-handed needling (Fig. 3; attempt a, d, e, h) and superficial (Fig. 3; target a, b, e, f) versus deep (Fig. 3; 


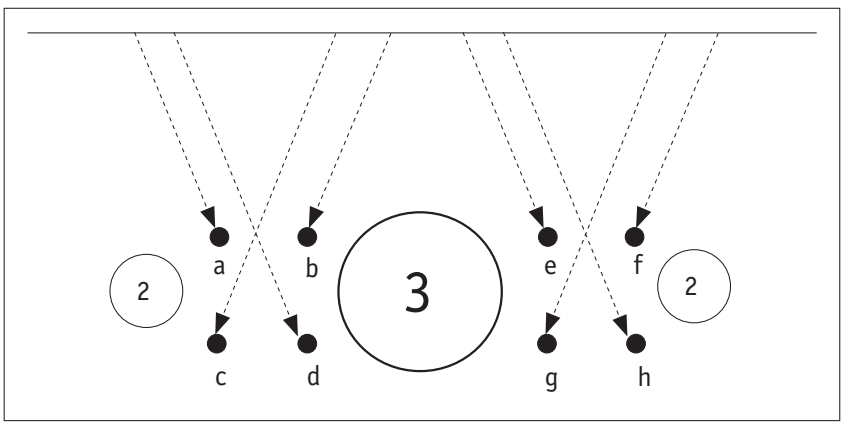

Fig. 3. Designated inserting trace. Arrows, designated inserting traces; dark spots, transverse section of 21-gauge needles mimicking "small nodules"; hollow circle 2, drinking straws mimicking "vessels"; hollow circle 3, urine tube mimicking "trachea." Dark spots in $a, b$, $e$, and $f$ represent superficial targets. Dark spots in $c, d, g$, and $h$ represent deep targets. Residents were instructed to puncture $b, c, f$, and $\mathrm{g}$ with right-handed needling and $\mathrm{a}, \mathrm{d}, \mathrm{e}$, and $\mathrm{h}$ with left-handed needling.

target $c, d, g, h)$ target attempts were also recorded.

Statistical analysis was conducted using SPSS Statistics 17.0 software (SPSS Inc., Chicago, IL, USA) to perform the Wilcoxon signed-rank test comparing success rates between pre-training and post-training, left-handed needling and right-handed needling, and superficial and deep target attempts. Statistical significance was noted when $p$ values were $<0.05$.

\section{RESULTS}

All of the materials for the phantom cost less than CNY 10 (approximately \$1.5) at a local store. Phantom assembly required approximately 10 minutes. On ultrasonography, the tofu exhibited heterogeneous hypoechogenicity, which mimicked thyroid tissue. The drinking straws filled with coupling gel exhibited an echogenicity in the center surrounded by a hyperechoic wall, which is similar to vessels. The urine tube filled with air exhibited hyperechogenicity with a long shadow that was similar to the trachea. The needles appeared as hyperechoic foci in the transverse section that mimicked "small nodules" (Fig. 4). With longitudinal orientation and alignment of the transducer, the 25-gauge needle was clearly observed as a linear hyperechogenicity on ultrasound images. The entire puncture procedure could be distinctly visualized. When the inserting needle touched the "small nodule," the procedure could be visualized on the screen, and resistance could be felt in the needle-holding hand. In addition, movement of the outside hub was observed. The 25-gauge puncture needle left a very faint track that almost disappeared when

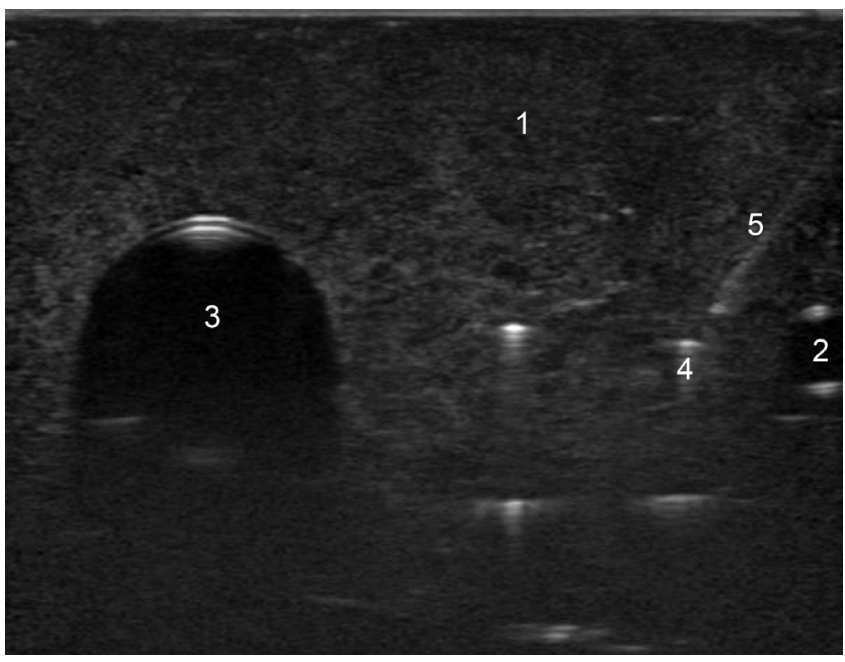

Fig. 4. Ultrasonography of phantom. 1, "thyroid" (tofu); 2, "vessel" (drinking straw); 3, "trachea" (urine tube); 4, "small nodule" (transverse section of 21-gauge needle); 5, 25-gauge needle.

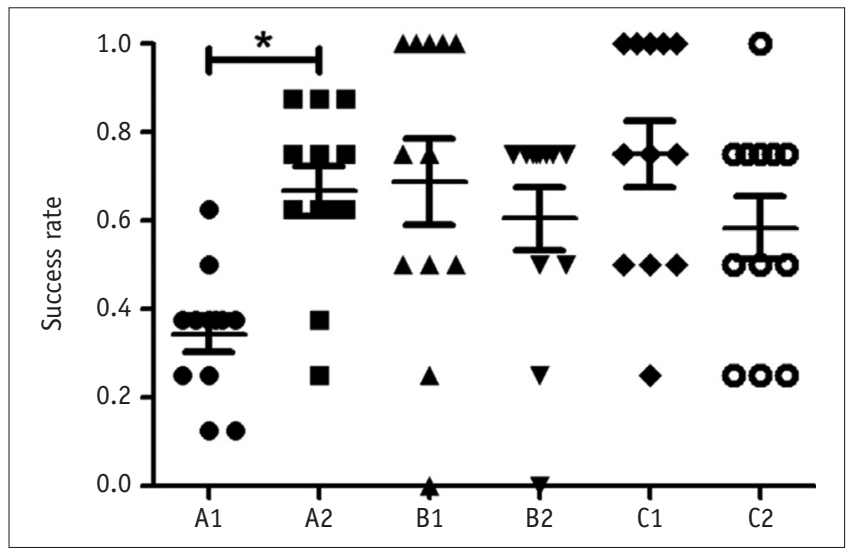

Fig. 5. Comparison of success rates between pre- and posttraining, superficial and deep targets, and right-handed and left-handed needling. A1, pre-training group; $A 2$, post-training group; B1, superficial target group; B2, deep target group; C1, righthanded needling group; C2, left-handed needling group. Each circle, square, triangle, or rhombus represents success rate of resident in corresponding group. Middle transverse line of symbol “王” represents mean value of success rate in group. Upper and lower lines represent $95 \%$ confidence intervals. ${ }^{*}$ Wilcoxon signed-rank test, $p<0.05$.

the needle was removed.

After training, the residents' success rate was significantly increased after training from $34.38 \pm 14.23 \%([2.8 \pm 1.1]$ / 8 targets $)$ to $66.7 \pm 19.5 \%$ ([5.3 \pm 1.6$] / 8$ targets $)$ $(p=0.003)$. All of the residents were right-handed. No significant difference was noted between right- and lefthanded needling $(p=0.101)$. No significant difference was noted between superficial and deep target attempts $(p=$ 0.458) (Fig. 5). 


\section{DISCUSSION}

The main practical training aspects of performing thyroid FNAB were achieved using the present tofu model: visualization and localization of the small nodule, retrieval, and confirmation of reaching the lesion. The tofu phantom model has several advantages. 1) All the materials are inexpensive and easily obtained. The entire phantom was produced for less than CNY 10 (approximately $\$ 1.5$ ) at a local store in China, which is slightly cheaper than the super-concentrated gelatin model (\$2-3) and much cheaper than a turkey breast model (approximately $\$ 20)(4,6)$. 2) The entire phantom is easily assembled in approximately 10 minutes, and no special skills are required. The tofu model is more readily available than the super-concentrated gelatin model, which takes 9-18 hours to ensure gelatin setting (6). 3) The echogenicity of tofu is more similar to the texture of thyroid. 4) The entire phantom includes "thyroid parenchyma," "trachea," and "vessels," which is similar to the structures in the neck. 5) We created small hyperechoic foci (only $0.81 \mathrm{~mm}$ in diameter) as targets, which increased the difficulty during practice sessions. Small nodules are more similar to microcalcifications, which often appear in malignant thyroid nodules (7). 6) The spatial distribution of the needles allows residents to practice the selection of adequate approaches for targeting a "small nodule" in different quadrants. Both hands can be used to practice handling the transducer and holding the needle in an alternate fashion, which is a practical skill needed for FNAB in humans. 7) When the needle touches the "small nodule," the operators can visualize it on the screen, and resistance can be felt in their hand. The movement of the hub can also confirm the success.

Twelve radiology residents were involved in this study. After training, success rates were significantly increased indicating that the tofu phantom was an effective method to improve their puncture accuracy in vitro. Although no significant differences were noted between right-handed and left-handed needling, most of the residents felt more comfortable when they used their right hand to hold the needle and left hand to hold the probe. All of the residents were right-handed and frequently used their right hand for subtle movements, and this finding was attributed to the fact that the probe required less subtle movement than the needle. The depth of target did not impede the residents; the residents reported no differences in difficulty between superficial and deep target attempts. Moreover, no significant differences in targeting success were noted between superficial and deep targets. However, deep targets are more difficult in humans given the long path, and the needle trace is often missing in images. The relative ease of the technique might explain why the needle trace was more easily observed in the phantom compared with human tissue. Therefore, we suggest that residents should still begin with targeting superficial lesions with their right hand (for a right-handed individual) when starting FNAB in humans after training.

Some limitations of our study should be noted. 1) The human neck poses anatomic limitations to ultrasoundguided puncturing due to its convexity and complex structure. Therefore, it is more difficult to place a probe flatly and get a whole-screen image in humans. In this study, the phantom was flat and an entire image could be more easily obtained. Furthermore, the phantom did not include "muscles" and "skin" in the "neck." All of these characteristics differ among humans. 2) While other model targets with more similarity to nodules have been reported, such as olives, berries, fish oil capsules, or cocktail onions (6), we used needles for puncturing because air surrounded the "nodules," inducing strong reflections after such targets were placed in the center of the tofu. Moreover, in the present study, the "small nodule" was not a point but rather a line. Therefore, we did occasionally observe movement in the external hub while failing to observe whether the 25-gauge needle touched the "small nodule" in the realtime image due to the 25-gauge needle reaching the 21-gauge needle in another section. We considered this scenario a target failure. 3 ) The model does not sufficiently maintain the integrity of the entire procedure of fine needle aspiration. Only puncturing the "small nodule" was practiced. Training in aspiration was not offered, as the puncturing needle could not aspirate the "small nodule" that was represented by needles.

In summary, the consistency and echotexture of the tofu phantom are quite similar to the actual human thyroid and surrounding tissues. This model is cost-effective, easily attainable, and effective for training residents in ultrasound-guided fine needle thyroid nodule targeting punctures in vitro.

\section{Conflicts of Interest}

The authors have no financial conflicts of interest. 


\section{Acknowledgments}

The authors thank Ting-Ting Zhong, Guang-Ting Chai, and Shi-Yi Xu for their help with photography and preparing the materials.

\section{ORCID}

\section{Xue-Mei Wang}

https://orcid.org/0000-0002-8192-1068

Yun-Fei Zhang

https://orcid.org/0000-0001-9064-3314

\section{REFERENCES}

1. Shin JH, Baek JH, Chung J, Ha EJ, Kim JH, Lee YH, et al.; Korean Society of Thyroid Radiology (KSThR) and Korean Society of Radiology. Ultrasonography diagnosis and imagingbased management of thyroid nodules: revised Korean Society of Thyroid Radiology consensus statement and recommendations. Korean J Radiol 2016;17:370-395
2. Lee YH, Baek JH, Jung SL, Kwak JY, Kim JH, Shin JH; Korean Society of Thyroid Radiology (KSThR); Korean Society of Radiology. Ultrasound-guided fine needle aspiration of thyroid nodules: a consensus statement by the Korean Society of Thyroid Radiology. Korean J Radiol 2015;16:391-401

3. Gresens AA, Britt RC, Feliberti EC, Britt LD. Ultrasound-guided breast biopsy for surgical residents: evaluation of a phantom model. J Surg Educ 2012;69:411-415

4. Larrison M, DiBona A, Hogg DE. Low-cost phantom for stereotactic breast biopsy training. AJR Am J Roentgenol 2006;187:972-974

5. Quinn AD, Smiddy PF, Duggan M, Murphy J, Molloy M. Technical report: a training phantom for stereotactic breast biopsies. Clin Radiol 1997;52:149-150

6. Abraham D. A method using superconcentrated gelatin and a novel phantom suspension system for ultrasound-guided thyroid biopsy training. Thyroid 2014;24:1662-1663

7. Na DG, Baek JH, Sung JY, Kim JH, Kim JK, Choi YJ, et al. Thyroid imaging reporting and data system risk stratification of thyroid nodules: categorization based on solidity and echogenicity. Thyroid 2016;26:562-572 